In Crescendo, 2017; 8(2): 291-305

Fecha de recepción: 31 de mayo del 2017

Fecha de aceptación: 2 de noviembre del 2017

\title{
NIVEL DE CONOCIMIENTO, CONSUMO Y ACEPTACIÓN DE ALIMENTOS TRANSGÉNICOS EN POBLADORES DEL SECTOR SAN ANDRÉS, TRUJILLO 2016
}

\author{
LEVEL OF KNOWLEDGE, CONSUMPTION AND ACCEPTANCE \\ OF TRANSGENIC FOOD IN SETTLEMENTS OF \\ THE SECTOR SAN ANDRÉS, TRUJILLO 2016
}

\begin{abstract}
Juan Carlos Rodríguez Soto ${ }^{1}$, Marisol Contreras Quiñones ${ }^{2}$, Carmen Brigitte Espinoza Amaya ${ }^{3}$ Eldy Sarita Miranda Gallac
\end{abstract}

\section{RESUMEN}

El presente estudio se realizó con el objetivo de determinar el nivel de conocimiento, consumo y aceptación de alimentos transgénicos en pobladores de la Etapa I del Sector San Andrés del distrito de Trujillo durante los meses de marzo a junio del año 2016. La metodología es cualitativa y descriptiva cuasi experimental. La muestra total estuvo conformada por 86 viviendas donde se entrevistaron a personas mayores de edad de diferente género residentes en San Andrés. Se aplicó el instrumento de colección de datos que contuvo preguntas acerca del conocimiento, consumo y aceptación hacia los alimentos transgénicos. Con los datos se procedió a desarrollar gráficos y tablas que posteriormente fueron analizadas. Los resultados nos muestran un nivel de conocimiento bajo, aun cuando el nivel de consumo es elevado. Además la población muestra un nivel de aceptación bajo a los productos transgénicos.

PALABRAS CLAVE: Transgénico, nivel de conocimiento, consumo, aceptación.

1 Magister egresado de la Universidad Católica Los Ángeles de Chimbote.juanca_113@hotmail.com

2 Magister, docente de la Universidad Católica Los Ángeles de Chimbote.maryt_27@hotmail.com

3 Alumnas de Ciencias de la Salud de la Universidad Nacional de Trujillo. 


\section{ABSTRACT}

The present study was carried out with the objective of determining the level of knowledge, consumption and acceptance of transgenic foods in residents of Stage I of the San Andres Sector of the district of Trujillo during the months of March to June of the year 2016. The methodology is qualitative and descriptive quasi-experimental. The total sample consisted of 86 dwellings where elderly people of different genres residing in San Andrés were interviewed. The data collection instrument was applied, containing questions about knowledge, consumption and acceptance of transgenic foods. With the data we proceeded to develop graphs and tables that were later analyzed. The results show a low level of knowledge, even though the level of consumption is high. In addition, the population shows a low level of acceptance of transgenic products.

KEY WORDS: Transgenic, level of knowledge, consumption, acceptance.

\section{INTRODUCCIÓN}

La producción de alimentos transgénicos ha aumentado exponencialmente en los últimos años, lo que ha dado lugar a un consumo cada vez mayor. El acceso a los alimentos sanos y nutritivos, fundamental para asegurar el derecho a la alimentación, se ve por ende influenciado cada vez más por los transgénicos, relacionados cada vez más con aumentos de la productividad y variaciones en la calidad de los alimentos. ${ }^{1}$ Los alimentos genéticamente modificados conocidos como transgénicos pueden ser plantas, animales o microorganismos a los que se ha alterado el genoma o incluso aquellos que contienen algún derivado de los mismos. ${ }^{2}$

Los alimentos transgénicos son uno de los productos fundamentales de la evolución de la biotecnología de tercera y cuarta generación, originada desde mediados del siglo XX. Consisten en la manipulación del ácido desoxirribonucleico (ADN) y el análisis de la secuencia de los nucleótidos del ADN y el conjunto de las proteínas. Este avance de la biotecnología permite modificar, eliminar o insertar genes dentro del ADN de un ser vivo de la misma especie o de otra con uno u otro fin. Eso se conoce como manipulación genética, lo que da lugar a los organismos modificados genéticamente $(\mathrm{OMG}){ }^{1}{ }^{1}$

Sin embargo los potenciales riesgos a los que nos podríamos ver expuestos con los alimentos genéticamente modificados (AGM) y que son el fundamento de organizaciones ecologistas que rechazan la utilización y consumo de AGM, no han sido debidamente rebatidos y algunos estudios consideran el desarrollo de alergias, la resistencia a los antibióticos, la pérdida o modificación del valor nutricional de los 
alimentos, la presencia de compuestos tóxicos, la aparición de enfermedades nuevas y no tratables, además del daño a las especies silvestres de plantas. ${ }^{3}$

Sin embargo ello no ha detenido el avance mundial, pues años tras año se presenta un crecimiento exponencial del área cultivada con transgénicos. En el año 1996 se tenían sólo 2,3 millones de hectáreas, y al año 2000, se sembraron 44,2 millones de hectáreas con cultivos transgénicos, de los cuales el 74\% del área plantada (32,7 millones de has.) corresponde a cultivos resistentes a herbicidas con cultivos de soya, maíz, algodón, papa, arroz, sorgo, canola, caña de azúcar, tabaco, tomate, entre otros. ${ }^{4}$

Los cultivos transgénicos están muy concentrados en apenas 6 países, en unos pocos cultivos y en unas pocas características. Aunque hay muchas plantas transgénicas, sólo unas pocas se cultivan ${ }^{4}$. Estados Unidos (63\%), Argentina (21\%), Canadá (6\%), China (4\%), Brasil (4\%) y Sudáfrica (1\%) representan el 99\% de la superficie plantada con transgénicos en 2003. Los transgénicos se cultivan en 7 países industrializados (Estados Unidos, Canadá, Australia, España, Alemania, Rumania y Bulgaria) y en 11 países en desarrollo (Argentina, China, Suráfrica, México, Indonesia, Brasil, India, Uruguay, Colombia, Honduras y Filipinas). ${ }^{4}$ Actualmente existen en el mercado cuatro cultivos: maíz, algodón, soya y canola, que se siembran a nivel comercial en varios países: Estados Unidos, Argentina, Brasil, Canadá, India, China, Paraguay y Sudáfrica.

El movimiento de tierras dedicadas al cultivo de transgénicos nos muestra escenarios diferentes, China en el 2000 superó los 2,8 millones de hectáreas de algodón transgénico (58\% del cultivo nacional de algodón). Suráfrica el mismo año sembró 400.000 hectáreas, un 33\% más que en 2002 y un 1\% del total mundial (maíz Bt, algodón y soja). En Australia disminuyó la superficie cultivada, que fue de sólo 100.000 hectáreas de algodón transgénico. India continua plantando algodón Bt, llegando a 100.000 hectáreas en 2003. Uruguay supera ya plantaciones de 60.000 hectáreas de soja y maíz Bt, y Rumania supera las 70.000 hectáreas de soja transgénica. Argentina en el año 2003 plantó 13,9 millones de hectáreas, un 3\% más que en 2002 y un 21\% del total mundial (maíz Bt, y casi el 100\% de la superficie de soja). Brasil, inició en el 2003 la siembra de soja transgénica legalmente por primera vez (ya se importaban semillas de soja transgénica de contrabando, procedentes de Argentina). ${ }^{4}$

En el Perú existe a la fecha existe una ley, la № 29811, que establece la moratoria al ingreso y producción de transgénicos en el territorio nacional por 10 
años ${ }^{6}$, restringiendo su manipulación solo con fines de investigación; sin embargo, a mediados del 2011 se detectó la comercialización de productos con ingredientes transgénicos en las tiendas comerciales. La Asociación Peruana de Consumidores y Usuarios (ASPEC) publicó una lista de alimentos que contienen organismos genéticamente modificados. ${ }^{7}$

En base a lo expuesto la pree investigación se planteó el siguiente problema de investigación: ¿Cuál es el nivel de conocimiento, consumo y aceptación de alimentos transgénicos en pobladores del sector San Andrés, Trujillo 2016?

Planteándonos como objetivo general el determinar el nivel de conocimiento, consumo y aceptación de alimentos transgénicos en pobladores del sector San Andrés, Trujillo 2016. Teniendo como objetivos específicos determinar el nivel de conocimiento de los alimentos transgénicos por género, determinar el nivel de consumo de alimentos transgénicos por género, así mismo determinar el nivel de aceptación de los alimentos transgénicos por género.

\section{MATERIAL Y MÉTODO}

La presente investigación, de acuerdo a la clasificación propuesta por Hernánde $z^{8}$, se ajusta a un tipo cualitativo y de diseño descriptivo cuasi experimental.

La muestra lo constituyeron 86 viviendas, se entrevistaron a personas con mayoría de edad y residentes en la Etapa I del Sector San Andrés del distrito de Trujillo, de domicilios diferentes, los mismos que fueron seleccionados al azar y con participación consentida.

La técnica realizada fue la entrevista y el instrumento fue un cuestionario con 11 preguntas de los temas de conocimiento (4 preguntas), consumo ( 3 preguntas) y aceptación (3 preguntas) de los alimentos de origen transgénico, y una pregunta de rango de edad. Las entrevistas se realizaron entre los meses de marzo a junio del 2016.

Para aplicar el instrumento se socializó los objetivos de la investigación y se buscó obtener el consentimiento voluntario de los entrevistados para el desarrollo de la presente investigación. Se siguió lo propuesto por Polit ${ }^{9}$ en referencia a procedimientos éticos fundamentales.

Una vez terminada la recolección de datos, estos se analizaron estadísticamente con la ayuda del programa estadístico Statgraphics Plus 5.1. obteniéndose medidas de tendencia central y con los mismos datos se elaboraron gráficos para la mejor presentación y discusión de los mismos. 


\section{RESULTADOS}

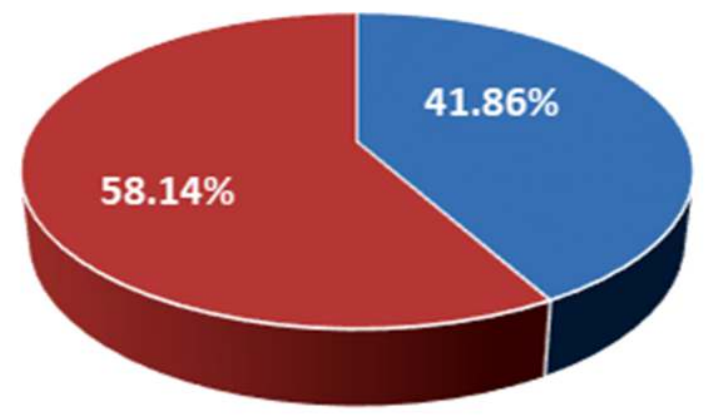

- masculino - femenino

Gráfico 1. Distribución porcentual de la muestra según su género.

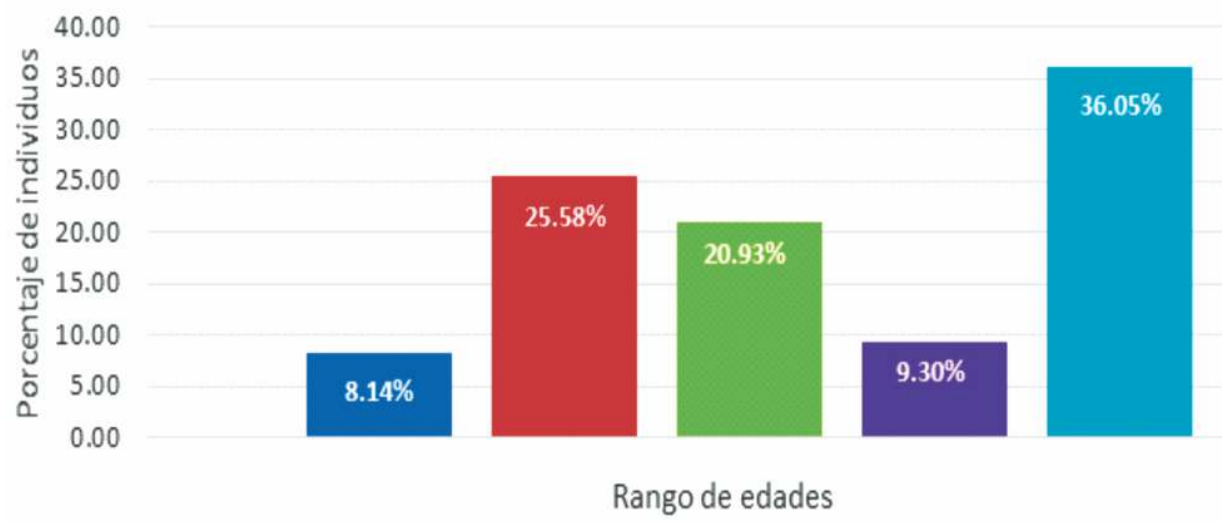

-Menor a 20 años $\square 20-29 \square 30-39 \square 40-49 \square$ Mayor a 50 años

Gráfico 2. Distribución porcentual de la muestra según rango de edad en años. 
Juan Rodríguez Soto, Marisol Contreras Quiñones, Carmen Espinoza Amaya, Eldy Miranda Gallac

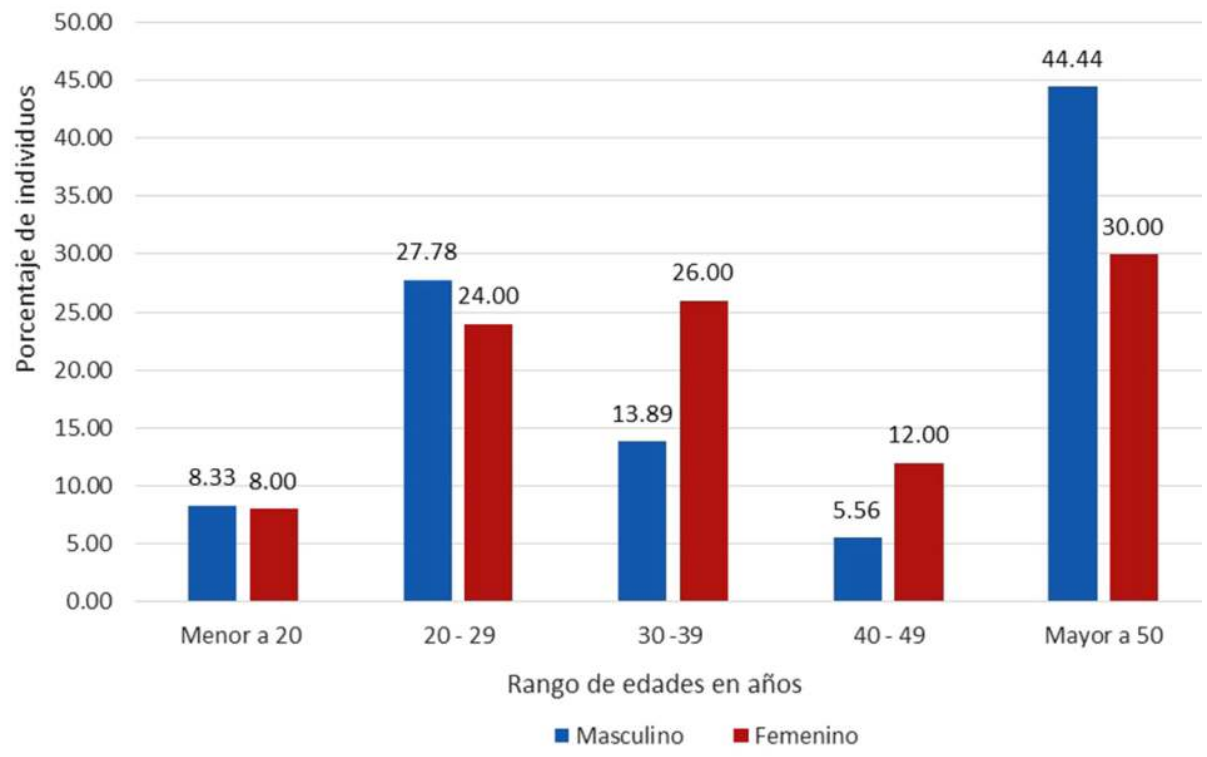

Gráfico 3. Distribución porcentual de la muestra según rango de edad en años y género.

Tabla 1

DISTRIBUCIÓN PORCENTUAL DE LA MUESTRA FRENTE A LA MANERA DE OBTENER INFORMACIÓN ACERCA DE LOS ALIMENTOS QUE CONSUME

\begin{tabular}{lrrrrrc}
\hline & $\%$ & Total & $\%$ & Masculino & $\%$ & Femenino \\
\hline Libros & 8,14 & 7 & 5,56 & 2 & 10,00 & 5 \\
Revistas & 20,93 & 18 & 22,22 & 8 & 20,00 & 10 \\
Etiquetas & 6,98 & 6 & 8,33 & 3 & 6,00 & 3 \\
TV & 27,91 & 24 & 22,22 & 8 & 32,00 & 16 \\
Supermercados & 10,47 & 9 & 5,56 & 2 & 14,00 & 7 \\
Amigos & 1,16 & 1 & 2,78 & 1 & 0,00 & 0 \\
Otros & 24,42 & 21 & 33,33 & 12 & 18,00 & 9 \\
\hline Total & 100,00 & 86 & 100,00 & 36 & 100,00 & 50 \\
\hline
\end{tabular}

Fuente: Encuesta aplicada a los pobladores de la Etapa I Sector San Andrés, Trujillo - Marzo a Junio del 2016. 
Tabla 2

DISTRIBUCIÓN PORCENTUAL DE LA MUESTRA

FRENTE AL CONOCIMIENTO DE LO QUE ES

UN PRODUCTO TRANSGÉNICO

\begin{tabular}{lrcrcrc}
\hline & $\%$ & Total & $\%$ & Masculino & $\%$ & Femenino \\
\hline Mucho & 2,33 & 2 & 5,56 & 2 & 0,00 & 0 \\
Sí & 27,91 & 24 & 38,89 & 14 & 20,00 & 10 \\
Algo & 24,42 & 21 & 33,33 & 12 & 18,00 & 9 \\
Poco & 18,60 & 16 & 8,33 & 3 & 26,00 & 13 \\
Nada & 26,74 & 23 & 13,89 & 5 & 36,00 & 18 \\
\hline Total & 100 & 86 & 100 & 36 & 100 & 50 \\
\hline
\end{tabular}

Fuente: Encuesta aplicada a los pobladores de la Etapa I Sector San Andrés, Trujillo - Marzo a Junio del 2016.

Tabla 3

DISTRIBUCIÓN PORCENTUAL DE LA MUESTRA FRENTE

AL CONOCIMIENTO DE LA FINALIDAD DEL

MEJORAMIENTO VEGETAL Y ANIMAL.

\begin{tabular}{lcccccc}
\hline & $\%$ & Total & $\%$ & Masculino & $\%$ & Femenino \\
\hline Nutrición & 23,26 & 20 & 22,22 & 8 & 24,00 & 12 \\
Medicinal & 10,47 & 9 & 8,33 & 3 & 12,00 & 6 \\
Sabor & 1,163 & 1 & 2,78 & 1 & 0,00 & 0 \\
Apariencia & 17,44 & 15 & 22,22 & 8 & 14,00 & 7 \\
Económicos & 40,70 & 35 & 27,78 & 10 & 50,00 & 25 \\
Otros & 6,98 & 6 & 16,67 & 6 & 0,00 & 0 \\
\hline Total & 100 & 86 & 100 & 36 & 100 & 50 \\
\hline
\end{tabular}

Fuente: Encuesta aplicada a los pobladores de la Etapa I Sector San Andrés, Trujillo - Marzo a Junio del 2016. 
Juan Rodríguez Soto, Marisol Contreras Quiñones, Carmen Espinoza Amaya, Eldy Miranda Gallac

Tabla 4

DISTRIBUCIÓN PORCENTUAL DE LA MUESTRA FRENTE

AL CONSUMO DE UNA LISTA DE PRODUCTOS

CON INGREDIENTES TRANSGÉNICOS

\begin{tabular}{ccccccc}
\hline & $\%$ & Total & $\%$ & Masculino & $\%$ & Femenino \\
\hline $\begin{array}{c}\text { Soya Andina - } \\
\text { Soya Sta. Catalina } \\
\text { Quaker "Q-vital" } \\
\quad 18,75\end{array}$ & 36 & 14,81 & 12 & 21,62 & 24 \\
$\quad$ Quinua & 34,38 & 66 & 37,04 & 30 & 32,43 & 36 \\
$\begin{array}{c}\text { Cuates Picantes/ } \\
\text { Karinto }\end{array}$ & 7,29 & 14 & 9,88 & 8 & 5,41 & 6 \\
$\begin{array}{c}\text { Salchichas San } \\
\text { Fernando }\end{array}$ & 9,38 & 18 & 12,35 & 10 & 7,21 & 8 \\
Maicena Negrita & 15,10 & 29 & 11,11 & 9 & 18,02 & 20 \\
Angel Flakes & 15,10 & 29 & 14,81 & 12 & 15,32 & 17 \\
\hline Total & 100,00 & 192 & 100,00 & 81 & 100,00 & 111 \\
\hline
\end{tabular}

Nota: Los encuestados tuvieron múltiples respuestas.

Fuente: Encuesta aplicada a los pobladores de la Etapa I Sector San Andrés, Trujillo - Marzo a Junio del 2016.

Tabla 5

DISTRIBUCIÓN PORCENTUAL DE LA MUESTRA FRENTE AL

FACTOR QUE INFLUYE PREPONDERANTEMENTE EN EL CONSUMO DE LA LISTA DE ALIMENTOS DE LA TABLA 4

\begin{tabular}{lcccccc}
\hline & $\%$ & Total & $\%$ & Masculino & $\%$ & Femenino \\
\hline Valor Nutricional & 53,49 & 46 & 38,89 & 14 & 64,00 & 32 \\
Sabor & 23,26 & 20 & 33,33 & 12 & 16,00 & 8 \\
Precio & 6,98 & 6 & 2,78 & 1 & 10,00 & 5 \\
Calidad & 9,30 & 8 & 8,33 & 3 & 10,00 & 5 \\
Otro & 6,98 & 6 & 16,67 & 6 & 0,00 & 0 \\
\hline Total & 100 & 86 & 100 & 36 & 100 & 50 \\
\hline
\end{tabular}

Fuente: Encuesta aplicada a los pobladores de la Etapa I Sector San Andrés, Trujillo - Marzo a Junio del 2016. 
Tabla 6

DISTRIBUCIÓN PORCENTUAL DE LA MUESTRA FRENTE A LA FRECUENCIA DE CONSUMO DE LOS ALIMENTOS

DE LA LISTA EN LA TABLA 4

\begin{tabular}{lcccccc}
\hline & $\%$ & Total & $\%$ & Masculino & $\%$ & Femenino \\
\hline A Diario & 50,00 & 43 & 36,11 & 13 & 60,00 & 30 \\
A 2 - 3 dias & 31,40 & 27 & 41,67 & 15 & 24,00 & 12 \\
A la Semana & 15,12 & 13 & 22,22 & 8 & 10,00 & 5 \\
Al mes & 2,33 & 2 & 0,00 & 0 & 4,00 & 2 \\
Casi Nunca & 1,16 & 1 & 0,00 & 0 & 2,00 & 1 \\
\hline Total & 100 & 86 & 100 & 36 & 100 & 50 \\
\hline
\end{tabular}

Fuente: Encuesta aplicada a los pobladores de la Etapa I Sector San Andrés, Trujillo - Marzo a Junio del 2016.

Tabla 7

DISTRIBUCIÓN PORCENTUAL DE LA MUESTRA FRENTE A LA PROCEDENCIA DE LA LISTA DE ALIMENTOS

DE LA TABLA 4.

\begin{tabular}{lrcrccc}
\hline & $\%$ & Total & $\%$ & Masculino & $\%$ & Femenino \\
\hline Mucho & 2,33 & 2 & 0,00 & 0 & 4,00 & 2 \\
Sí & 27,91 & 24 & 22,22 & 8 & 32,00 & 16 \\
Algo & 24,42 & 21 & 22,22 & 8 & 26,00 & 13 \\
Poco & 18,60 & 16 & 27,78 & 10 & 12,00 & 6 \\
Nada & 26,74 & 23 & 27,78 & 10 & 26,00 & 13 \\
\hline Total & 100 & 86 & 100 & 36 & 100 & 50 \\
\hline
\end{tabular}

Fuente: Encuesta aplicada a los pobladores de la Etapa I Sector San Andrés, Trujillo - Marzo a Junio del 2016. 
Juan Rodríguez Soto, Marisol Contreras Quiñones, Carmen Espinoza Amaya, Eldy Miranda Gallac

Tabla 8

DISTRIBUCIÓN PORCENTUAL DE LA MUESTRA FRENTE A LA POSICIÓN PERSONAL QUE TIENEN FRENTE AL CONSUMO Y COMERCIALIZACIÓN DE PRODUCTOS TRANSGÉNICOS

\begin{tabular}{lrcrccc}
\hline & $\%$ & Total & $\%$ & Masculino & $\%$ & Femenino \\
\hline Muy Favorable & 1,16 & 1 & 2,78 & 1 & 0,00 & 0 \\
Favorable & 18,60 & 16 & 27,78 & 10 & 12,00 & 6 \\
Indiferente & 27,91 & 24 & 33,33 & 12 & 24,00 & 12 \\
Desfavorable & 39,53 & 34 & 27,78 & 10 & 48,00 & 24 \\
No opina & 12,79 & 11 & 8,33 & 3 & 16,00 & 8 \\
\hline Total & 100 & 86 & 100 & 36 & 100 & 50 \\
\hline
\end{tabular}

Fuente: Encuesta aplicada a los pobladores de la Etapa I Sector San Andrés, Trujillo - Marzo a Junio del 2016.

Tabla 9

DISTRIBUCIÓN PORCENTUAL DE LA MUESTRA FRENTE A LA PREGUNTA SI CONSUMIRÍA UN PRODUCTO TRANSGÉNICO.

\begin{tabular}{lrrrrrr}
\hline & $\%$ & Total & $\%$ & Masculino & $\%$ & Femenino \\
\hline Mucho & 0 & 0 & 0,00 & 0 & 0,00 & 0 \\
Sí & 31,40 & 27 & 36,11 & 13 & 28,00 & 14 \\
Algo & 12,79 & 11 & 22,22 & 8 & 6,00 & 3 \\
Poco & 15,12 & 13 & 8,33 & 3 & 20,00 & 10 \\
Nada & 40,70 & 35 & 33,33 & 12 & 46,00 & 23 \\
\hline Total & 100 & 86 & 100 & 36 & 100 & 50 \\
\hline
\end{tabular}

Fuente: Encuesta aplicada a los pobladores de la Etapa I Sector San Andrés, Trujillo - Marzo a Junio del 2016. 
Tabla 10

DISTRIBUCIÓN PORCENTUAL DE LA MUESTRA FRENTE A LA SITUACIÓN DE ENTERARSE SI UNO DE LOS ALIMENTOS QUE VIENE CONSUMIENDO ES TRANSGÉNICO Y SI SEGUIRÍA CONSUMIÉNDOLO

\begin{tabular}{lrccccc}
\hline & $\%$ & Total & $\%$ & Masculino & $\%$ & Femenino \\
\hline Deja de Consumirlo & 50 & 43 & 63,89 & 23 & 40,00 & 20 \\
Lo Analizaría & 29,07 & 25 & 27,78 & 10 & 30,00 & 15 \\
No sabe & 5,81 & 5 & 0,00 & 0 & 10,00 & 5 \\
Otro & 15,12 & 13 & 8,33 & 3 & 20,00 & 10 \\
\hline Total & 100 & 86 & 100 & 36 & 100 & 50 \\
\hline
\end{tabular}

Fuente: Encuesta aplicada a los pobladores de la Etapa I Sector San Andrés, Trujillo - Marzo a Junio del 2016.

\section{DISCUSIÓN}

Desde su origen, los alimentos transgénicos han sido capaces de generar una enorme controversia por sus efectos positivos y negativos. Por un lado, es capaz de aumentar la productividad de la tierra y por otro las consecuencias pueden ser imprevisibles.

En el Gráfico 1 se muestra la distribución porcentual de la muestra según el género, donde el mayor porcentaje de entrevistados fueron del género femenino $(58,14 \%)$ frente al género masculino con un $41,86 \%$, esto probablemente se deba a que en la mayoría de viviendas la mujer es la que queda al cuidado casa y el varón sale a trabajar. Esto colaboró con nuestros resultados de consumo, toda vez que las respuestas fueron dadas en su mayoría por las féminas, que mayormente se encargan de las compras de los alimentos del hogar.

En el Gráfico 2 como en el Gráfico 3 se observan la distribución porcentual de los entrevistados según rango de edad, en el primero observamos la distribución sin tener en cuenta el género, observándose que el mayor porcentaje de entrevistados supera los 50 años de edad (36,05\%), seguidos con un 25,58\% por personas cuya edad se encuentra entre 20 a 29 años de edad. En el siguiente gráfico la distribución por rango de edad tiene en cuenta el género, y el rango donde se encuentran la mayor cantidad de entrevistadas mujeres es el de 30 a 39 años (30\%), mientras que en los varones es el rango de mayores de 50 años con un $44,44 \%$. 
En cuanto al nivel de conocimiento de los productos transgénicos en la Tabla 1 muestra que la mayoría de mujeres entrevistadas consiguen información de sus alimentos a través de la televisión (32\%) dejando como última opción las etiquetas (6\%); mientras que en el género masculino la información a través dela televisión como la de revistas ocupa el primer lugar de preferencia $(22,22 \%)$ dejando como última opción los consejos o información recibida de las amistades $(2,78 \%)$. La Tabla 2 responde al conocimiento que se tiene de un producto transgénico, teniendo que el 36\%de mujeres refiere un total desconocimiento y solo un 20\% refiere conocer acerca del tema; en el género masculino por el contrario el mayor porcentaje $(38,89 \%)$ refiere sí conocer del tema y solo un $13,89 \%$ señala no conocer del tema. En la Tabla 3 la distribución porcentual se refiere al conocimiento que poseen los entrevistados acerca de la finalidad que tienen los productos transgénicos, encontrándose que en la mayoría de mujeres (50\%) piensa que la finalidad es abaratar los costos de su producción, seguido por el $24 \%$ que piensa que la finalidad es mejorar el factor nutritivo. En el caso de los hombres la mayoría $(27,78 \%)$ coincide con las mujeres en referente al factor económico, seguido también de la idea de mejorar el valor nutricional (22,22\%) además de mejorar la apariencia del producto $(22,22 \%)$.

En cuanto al nivel de consumo de alimentos con componentes transgénicos, la Tabla 4 muestra el consumo de seis alimentos clasificados por ASPECPerú ${ }^{7}$ como productos con contenido transgénico, encontrándose un consumo regular de todos ellos en ambos géneros. La mayoría de mujeres (32,3\%) muestra un mayor consumo de quaker, al igual los varones muestran un mayor porcentaje $(37,0 \%)$ en dicho producto. En la Tabla 5 muestra las respuestas de los entrevistados en relación al porqué del consumo de la lista anterior (Tabla 4), a lo que la mayoría de mujeres y varones contestó que su consumo se basa al valor nutricional del producto (64\% y $38,89 \%$ respectivamente), seguido del factor sabor con un $16 \%$ y $33,33 \%$ respectivamente. La Tabla 6 responde a la frecuencia de consumo de la lista de la Tabla 4, teniendo que la mayoría de mujeres (60\%) consume a diario por lo menos uno de los productos de la lista en referencia, mientras que los varones en su mayoría consumen por lo menos uno de los productos cada 2 a 3 días $(41,67 \%)$. En la Tabla 7 se muestra la respuesta de los entrevistados a la pregunta si conocen la procedencia de dichos productos (Tabla 4), donde la mayoría de mujeres está en el grupo que sí conoce (32\%) o por lo menos conoce algo $(26 \%)$, mientras que la mayor parte de los varones se encuentra en las clasificaciones de conocer poco y conocer nada ( $27,78 \%$ en ambas). 
En Norte América, donde los cultivos y alimentos transgénicos están ya muy presentes, algunos científicos empiezan a pedir un mayor análisis de los organismos modificados genéticamente en relación con sus posibles efectos sobre la salud. Es el caso del Comité de Identificación y Asesoramiento sobre los Efectos No Deseados de los Alimentos Modificados Genéticamente en los Humanos creado por la Academia Nacional de Ciencias que en su informe de julio de 2004 hace recomendaciones para mejorar la evaluación de riesgo previa a la comercialización y el seguimiento una vez introducido en el mercado. ${ }^{10}$

En referencia al nivel de aceptación de productos transgénicos la Tabla 8 hace referencia a la posición del entrevistado en cuanto al consumo y comercialización de productos transgénicos, frente a ello la mayoría de mujeres (48\%) tuvo una opinión desfavorable, y los varones en su mayoría (33,33\%) se mostraron indiferentes al consumo. La Tabla 9 responde a la pregunta si el entrevistado estaría dispuesto a un consumo regular de algún producto transgénico, a lo que la mayoría de mujeres $(46 \%)$ respondió que no, mientras que la mayoría de varones (36,11\%) dijo que sí estaría dispuesto. En la Tabla 10 se le hizo la pregunta al entrevistado si se enteraría que usted está consumiendo algún producto transgénico que es lo que haría, a lo que la mayoría de mujeres y varones respondió dejaría de consumirlo ( $40 \%$ y $63,89 \%$ respectivamente).

En América del Sur existen escasos estudios sobre la aceptabilidad de alimentos transgénicos (AT). En Brasil, se determinó que 53\% de los encuestados había escuchado hablar de biotecnología, mientras la disposición a comprar AT se correlacionó con el lugar de residencia, ingreso y educación. En Argentina e independientemente del género del encuestado, se determinaron mayor aceptación hacia AT que beneficien la salud o el medio ambiente respecto de AT de menores precios. En Santiago, Chile, determinaron un escaso conocimiento de la biotecnología y de los AT, existiendo una negativa percepción pública del uso de alimentos transgénicos. ${ }^{11,12,13,14}$

Numerosos estudios dan cuenta de la preferencia del consumidor por alimentos libres de manipulación genética y rechazo hacia los alimentos transgéni$\cos (\mathrm{AT}){ }^{15,16,17,18,19}$ No obstante, algunos consumidores pueden ser indiferentes a esta condición (Townsend, 2006). La resistencia a la introducción de modificaciones genéticas $(\mathrm{MG})$ no es universal. Las actitudes respecto a la biotecnología dependen del organismo en el cual se aplica y el tipo de modificación realizada. Las modificaciones genéticas en plantas o microorganismos son más aceptadas 
que las modificaciones en animales. Además, los consumidores europeos se preocupan del consumo indirecto de este tipo de alimentos, es decir el uso de AT en alimentación animal. Asimismo, es relativamente más aceptada una MG para reducir el uso de pesticidas que las MGs para caracteres que permitan reducir los costos de producción o, si la modificación además presenta beneficios para el consumidor. ${ }^{14}$

\section{CONCLUSIONES}

- El nivel de conocimiento de alimentos transgénicos por parte delos pobladores de la Etapa I del Sector de San Andrés del distrito de Trujillo es bajo.

- El nivel de consumo de alimentos contemplados como transgénicos según ASPEC-Perú por parte delos pobladores de la Etapa I del Sector de San Andrés del distrito de Trujillo es alto.

- El nivel de aceptación de alimentos transgénicos por parte delos pobladores de la Etapa I del Sector de San Andrés del distrito de Trujillo es bajo.

\section{REFERENCIAS BIBLIOGRÁFICAS}

1. Herrero S. Los alimentos transgénicos como bienes públicos globales. Rev. Suma Neg. 2014; 5(10): 59-66.

2. MG (2013). La historia de Monsanto. Recuperado el 15 de febrero de 2014 de http://www.monsanto.com/global/ar/quienessomos/pages/historia-global.aspx

3. Reyes M, Rozowski J. Alimentos transgénicos. Rev. Chil. Nutr. 2003; 30(1):21-26.

4. Alonso G. En Colombia estamos consumiendo soya transgénica. 2000. Programas de Semillas de la Fundación Swissaid.

5. Massieu Y. Cultivos y alimentos transgénicos en México. Rev. Argumentos. 2009; 22 (59): 217-243.

6. Gutiérrez A. Segundo reporte nacional sobre la implementación del protocolo de Cartagena sobre la bioseguridad de la biotecnología. Ministerio del Ambiente. 2013. www.minam.gob.pe

7. La República. Recuperado 15 de febrero del 2016 de http://arepublica.pe/17-04-2011/conozca-la-lista-de-productos-con-transgenicos

8. Hernández R, Fernández C, Baptista P. Metodología de la Investigación. 4 ed. México. Edit. Mc Graw-Hill Interamericana. 2006.

9. Polit D, Hungler B. Investigación científica en Ciencias de la Salud. 6 ed. México. Mc GrawHill Interamericana. 2000.

10. Spendeler L. Organismos modificados genéticamente: una nueva amenaza para la seguridad alimentaria. Rev. Esp. Salud Pública. 2005; 9(2):271-282. 
11. Oda L, Soares B. Genetically modified foods: economics aspects and public acceptance in Brazil. Trends in Biotechnology. 2000; 5: 188-190.

12. Mucci A, Hough G. Perceptions of genetically modified foods by consumers in Argentina. Food Quality and Preference. 2004; 15: 43-51.

13. Gil L, Martínez V, Irarrázabal C, Martínez C. Aceptación pública de la biotecnología y de los alimentos transgénicos. Rev. Ambiente y Desarrollo. 2001; 17:52-59.

14. Schnettler B, Sepúlveda O, Ruiz D. Aceptación diferenciada de alimentos transgénicos de origen vegetal y animal en la Región Araucanía Chile. Rev. Cienc Inv. Agr. 2008; 35 (2): 169 180.

15. Ganieri R, Chern W, Hahn D. A continuum of consumer attitudes toward genetically modified foods in the United States. Journal of Agricultural Resource Economics. 2006; 31: 129. 149 .

16. Ho R, Vermeer E, Zhao J. Biotechnology and food safety in China: Consumere acceptance or resistance. Development and Change. 2006; 37:227-253.

17. Larue B, West C, Gendron C, Lambert R. Consumer response to functional foods produced by conventional, organic, or genetic manipulation. Agribusiness. 2004; 20: 155-166.

18. Lusk J, Roosen J, Fox J. Demand for beef from cattle administered growth hormones or fed genetically modified cord; a comparison of consumers in France, Germany, the United Kingdom and the United States. American Journal of Agricultural Economics. 2003; 85: 16-29.

19. Lusk J, Daniel M, Mark D, Lusk C. Alternative calibration and auction institutions for predicting consumer willingness to pay for nongenetically modified corn chips. JOurnal of Agricultural REsource Economics. 2001;26;40-57. 